\title{
Expression of glomerular extracellular matrix components in human diabetic nephropathy: decrease of heparan sulphate in the glomerular basement membrane
}

\author{
J.T. Tamsma ${ }^{1}$,J. van den Born ${ }^{4}$,J. A.Bruijn ${ }^{2}$, K.J.M. Assmann ${ }^{5}$, J.J. Weening ${ }^{6,7}$, J.H.M. Berden ${ }^{4}, J_{\text {. Wieslander }}^{8}$, \\ E.Schrama ${ }^{3}$, J.Hermans ${ }^{9}$, J.H. Veerkamp ${ }^{10}$, H.H.P.J.Lemkes ${ }^{1}$, F.J. van der Woude ${ }^{2}$ \\ Departments of ${ }^{1}$ Endocrinology, ${ }^{2}$ Pathology and ${ }^{3}$ Nephrology, University Hospital Leiden, The Netherlands, Departments of \\ ${ }^{4}$ Nephrology and ${ }^{5}$ Pathology, University Hospital Nijmegen, The Netherlands, ${ }^{6}$ Department of Pathology, University of Groningen, \\ The Netherlands, ${ }^{7}$ Department of Pathology, University of Amsterdam, The Netherlands, ${ }^{8}$ Department of Autoimmune Serology, \\ Statens Seruminstitut, Copenhagen, Denmark, ${ }^{9}$ Department of Medical Statistics, University of Leiden, The Netherlands, \\ ${ }^{10}$ Department of Biochemistry, University of Nijmegen, The Netherlands
}

Summary Diabetic nephropathy is characterized by albuminuria which proceeds to overt proteinuria. The highly negatively stained HS side chain of heparan sulphate proteoglycan (HSPG) is a major determinant of the charge-dependent permeability of the GBM. We set out to study the presence of HS and HSPG in the GBM of patients with diabetic nephropathy using newly developed monoclonal antibodies, and to compare HSPG expression to the expression of other previously investigated glomerular extracellular matrix compounds. Immunohistochemically, glomerular extracellular matrix components were analysed in 14 renal biopsies of patients with diabetic nephropathy and compared with those of normal control subjects. Monoclonal antibodies used were: JM403 against the HS side chain of GBM HSPG and JM72 against the HSPG-core protein. Also, a polyclonal antiserum (B31) against human GBM-HSPG-core protein was used. Additionally, antibodies were used against collagen types I, III, IV and against $\alpha 1$ (IV)NC, $\alpha 3($ IV)NC and fibronectin. Staining was scored for intensity and for staining pattern by four independent observers who had no previous knowledge of the sample origin. No glomerular staining was seen for collagen type I. Collagen type III was present in some diabetic nodules. Anti-collagen type IV showed a decreased GBM stain-

Received: 18 June 1993

and in revised form: 12 October 1993

Corresponding author: Dr. J.T.Tamsma, University Hospital Leiden, Building 1, C4-R, P.O. Box 9600, NL-2300 RC Leiden, The Netherlands

Abbreviations: HS: Heparan sulphate; GBM: glomerular basement membrane; HSPG: heparan sulphate proteoglycan; NC: noncollagenous globular domain; IDDM, insulin-dependent diabetes mellitus; NIDDM, non-insulin-dependent diabetes mellitus ing in patients with diabetic nephropathy $(p=0.04)$. With anti- $\alpha 1$ (IV)NC no changes in GBM staining intensity were observed; with anti- $\alpha 3$ (IV)NC brilliant GBM staining was seen in both groups. Increased mesangial staining ( $p=0.003$ ) was seen with anti-collagen type IV in biopsies with nodular lesions. No differences were observed for fibronectin although it was abundantly present in the mesangial area of biopsies from patients with diabetic nephropathy. In biopsies with mesangial expansion and in biopsies with diabetic nodules, we observed a decreased GBM $(p=0.001) \mathrm{HS}$ side chain staining (JM403) without changes in HSPGcore protein staining (JM72,B31). The HS staining pattern regularly changed from a linear to a more granular and irregular pattern. In patients with a creatinine clearance of more than $15 \mathrm{ml} / \mathrm{min}$, the intensity of GBM HS staining showed an inverse correlation with the rate of proteinuria $(r=-0.85, p=0.004)$, suggesting a functional relationship. The decreased HS staining in the GBM may reflect the potentially disrupted charge barrier in diabetic nephropathy. [Diabetologia (1994) 37:313-320]

Key words Diabetic nephropathy, heparan sulphate, heparan sulphate proteoglycan, glomerular basement membrane, extracellular matrix.
Changes in HSPG, normally present in the GBM and to a lesser extent in the mesangial matrix, have been implicated in the pathogenesis of diabetic nephropathy [1-11]. These macromolecules are formed by a central core protein to which HS chains are linked by a trisaccharide. Sulphate groups are covalently bound to the repeating disaccharides of HS, resulting in a high negative charge. This negative charge is predominantly localized in the peripheral domains of the glycosaminoglycan chains [12]. Due to its chemical structure, HSPG 
Table 1. Characteristics of the patients

\begin{tabular}{|c|c|c|c|c|c|c|c|c|}
\hline Patient & $\begin{array}{l}\text { Age } \\
\text { (years) }\end{array}$ & $\begin{array}{l}\text { Sex } \\
(\mathrm{m} / \mathrm{f})\end{array}$ & $\begin{array}{l}\text { Diabetes } \\
\text { type }\end{array}$ & $\begin{array}{l}\text { Diabetes dura- } \\
\text { tion (years) }\end{array}$ & $\begin{array}{l}\text { Diabetic } \\
\text { retinopathy }\end{array}$ & $\begin{array}{l}\text { Creatinine clear- } \\
\text { ance }(\mathrm{ml} / \mathrm{min})\end{array}$ & $\begin{array}{l}\text { Proteinuria/ml } \\
\text { clearance }^{c}(\mu \mathrm{g} / \mathrm{ml})\end{array}$ & $\begin{array}{l}\text { Histologi- } \\
\text { cal stage }\end{array}$ \\
\hline 1 & 69 & f & NIDDM & 5 & 0 & 41 & 102 & $\mathrm{~d}$ \\
\hline 2 & 71 & $\mathrm{f}$ & NIDDM & 5 & 0 & 87 & 62 & d \\
\hline 4 & 63 & $\mathrm{~m}$ & NIDDM & 0 & 0 & 65 & 85 & d \\
\hline 5 & 65 & $\mathbf{f}$ & NIDDM & 7 & b & 22 & 158 & $\mathrm{n}$ \\
\hline 6 & 69 & f & NIDDM & 11 & b & 1 & 69 & n \\
\hline 9 & 52 & f & NIDDM & 10 & p & 13 & 321 & $\mathrm{n}$ \\
\hline 10 & 51 & $\mathrm{f}$ & NIDDM & 12 & $\mathrm{p}$ & 25 & 111 & $\mathrm{n}$ \\
\hline 11 & 60 & f & NIDDM & 1 & b & 100 & 56 & $n$ \\
\hline 12 & 45 & $\mathrm{~m}$ & IDDM & 33 & $\mathrm{p}$ & 40 & 132 & $\mathrm{n}$ \\
\hline 13 & 61 & $\mathrm{f}$ & IDDM & 48 & p & 9 & 54 & $\mathrm{n}$ \\
\hline
\end{tabular}

${ }^{a}$ Characterized according to criteria of the WHO [19].

${ }^{b}$ Diabetic retinopathy [41]: background (b), proliferative (p), absent or not recognized $(0)$.

${ }^{c}$ Ratio of proteinuria corrected for creatinine clearance (see subjects, materials and methods)
${ }^{\mathrm{d}}$ Histological stage of the tissue sections showed either diffuse (d) or nodular (n) mesangial enlargement.

${ }^{\mathrm{e}}$ md: missing data

Table 2. Antibodies and their characterization

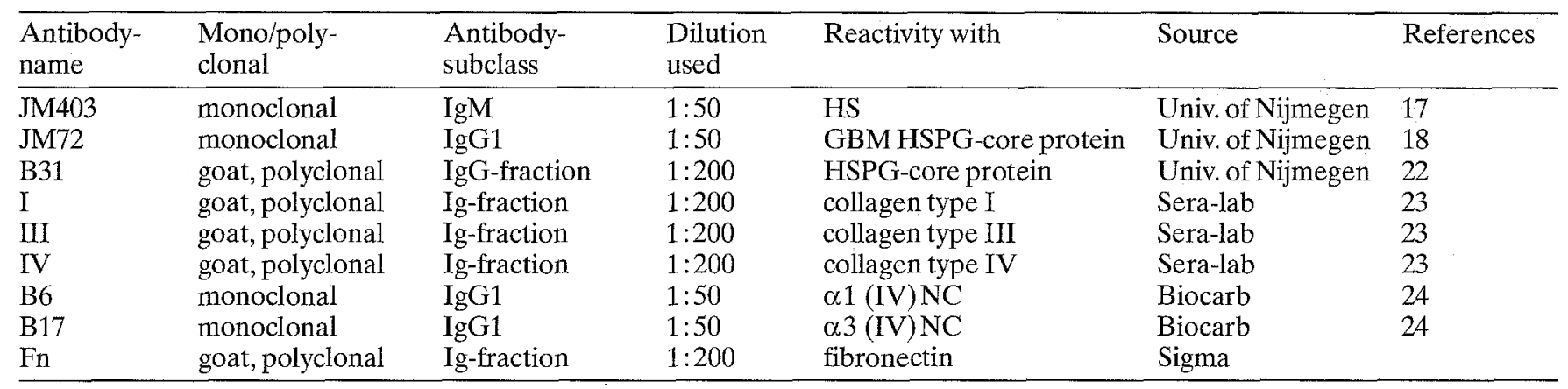

is an important component of the GBM. The molecule spreads out to occupy a large volume and attracts water molecules, thereby hydrating the GBM which prevents clogging of the filter. The highly negatively charged domains are major determinants of the charge-dependent permeability of the GBM $[13,14]$. In diabetes mellitus loss of HS from the GBM could lead to an enhanced filtration of negatively charged albumin. Moreover, in an in vitro model HS produced by glomerular epithelial cells was found to inhibit mesangial proliferation $[15$, 16]. Thus, mesangial expansion may at least partly be enhanced by a loss of glomerular HSPG in diabetic nephropathy. To study the presence of HS and HSPG in diabetic nephropathy more specifically, we used newly developed monoclonal antibodies with reactivity to human HS [17] and HSPG-core proteins [18]. Furthermore, HSPG expression was compared to the expression of other previously investigated glomerular extracellular matrix compounds.

\section{Subjects, materials and methods}

Screening of renal biopsies obtained from proteinuric diabetic patients was performed by a computer assisted search on coded files in three pathology departments. Twenty renal biopsies and four specimens obtained during autopsy were selected. Four biopsies showed other abnormalities; a crescentic glomerulonephritis in one and ischaemic alterations obscuring the diabeticlesions in three biopsies. Of the remaining 19 biopsies, one biopsy showed end-stage renal disease with total sclerosis of glomeruli, and three biopsies were too small to be evaluated properly. After reviewing the clinical data two more biopsies were excluded since the nephrotic syndrome present in these two patients showed a spontaneous remission. Fourteen biopsies revealed diabetic glomerulosclerosis and could be evaluated properly. These biopsies contained from 8 to more than 20 glomeruli of which $0 \%$ to $55 \%$ were sclerosed. The smallest number of open glomeruli studied was 5 (out of 9 glomeruli, 1 section). The clinical characteristics are summarized in Table 1. Patients were classified as having IDDM or NIDDM according to the criteria of the World Health Organisation [19]. In diabetic nephropathy, glomerular filtration rate and creatinine clearance show a strong inverse correlation with the percentage of occluded and globally sclerosed glomeruli $[20,21]$. We therefore corrected proteinuria $(\mathrm{g} / 24 \mathrm{~h})$ for the creatinine clearance $(\mathrm{m} 1 / \mathrm{min})$, yielding a ratio 
Table 3. Correlation and agreement between observers

\begin{tabular}{lllll}
\hline Observers & $n^{\mathrm{a}}$ & $r^{\mathrm{b}}$ & Kappa $^{\mathrm{c}}$ & $\%$ Agreement $^{\mathrm{d}}$ \\
\hline A vs B & 282 & 0.74 & 0.75 & 87 \\
A vs C & 265 & 0.69 & 0.59 & 80 \\
A vs D & 268 & 0.72 & 0.70 & 86 \\
B vs C & 266 & 0.72 & 0.69 & 85 \\
B vs D & 268 & 0.80 & 0.78 & 89 \\
C vs D & 254 & 0.66 & 0.56 & 77
\end{tabular}

${ }^{a} n$, number of observations based on 32 biopsies. The GBM intensity scores of all antibodies listed in Table 2 were used. Some small, later excluded sections were not scored by all observers.

${ }^{b} r$, correlation coefficient of the observations between two observers.

'Kappa, weighted Cohen's kappa.

d \% Agreement, percentage corresponding scores

which is expressed in $\mu \mathrm{g} / \mathrm{ml}$ (Table 1 ). This ratio of proteinuria reflects the protein loss in still-functioning glomeruli. The control group consisted of eight biopsies obtained post-mortem from organ donors before transplantation. The donors did not have albuminuria and were of similar age as the patients (mean 57 years, range $45-71$ years).

Antibodies. The composition of the extracellular matrix was analysed using monoclonal and polyclonal antibodies (Table 2). The various antibodies used with reactivity against $H S$ and HSPG-core protein have been previously described $[17,18]$. A monoclonal antibody (JM403) raised against rat glomerular HSPG, carrying the IgM isotype, reacted also with human HS. Furthermore, IgG1 monoclonal antibodies (JM72) and goat polyclonal antibodies (B31) [22] have been raised against human GBM-HSPG. These antibodies demonstrate specific reactivity to human GBM HSPG-core protein [18]. Affinity purified goat anti-human fibronectin antibodies were purchased from Sigma, (St. Louis, Mo., USA; cat. no. F-1509). Since the expression of the various collagen molecules and even of the collagen IV subchains can vary in diabetic nephropathy, several anti-collagen antibodies [23] were used. Goat anti-collagen type I, III, IV antibodies (Sera-lab, Crawley Down, Sussex, UK; cat: 1310-01, lot nr. F9-S389; cat: 1330-01, lot nr. F9-S409; cat: 1340-01, lot nr. F9S419, respectively) were raised against pepsin digested human and bovine placental collagens. Monoclonal antibodies against the non-collagenous domain of $\alpha 1(\mathrm{IV})$ and $\alpha 3(\mathrm{IV})$ were produced as described previously [24]. Rabbit anti-goat IgG and fluorescein isothiocyanate (FITC) conjugated goat anti-rabbit antibodies were raised in our laboratory using standard techniques. Rabbit anti-goat IgG antibodies (dilution 1: 75) followed by FITC conjugated goat anti-rabbit antibodies (dilution 1: 20) were used as the secondary layer for collagen type I, III, IV, fibronectin and B31. JM403 was visualized with FITC conjugated goat anti-mouse IgM (Nordic, Tilburg, The Netherlands; dilution 1: 50). FITC conjugated goat anti-mouse Ig (Becton-Dickinson, Mountain View, Calif., USA; cat. no. 9031, dilution 1: 40) was used for JM72, B6, B17. To check the specificity of the incubation procedure, tissue sections were also incubated only with the second antibodies. All these controls remained negative, although the FITC conjugated goat anti-mouse IgM (Nordic, dilution 1: 50) showed a slightly positive anti-nuclear staining.

Immunohistological methods. Immunofluorescence was performed on frozen kidney tissue specimens which were kept at $-80^{\circ} \mathrm{C}$ using standard techniques. All slides were prepared by the same laboratory technician. The kidney tissue was sectioned at $4 \mu \mathrm{m}$ in a Reichert Jung 2800 cryostat at $-20^{\circ} \mathrm{C}$. The sections were stained with primary antibodies in $1 \%$ bovine serum al-
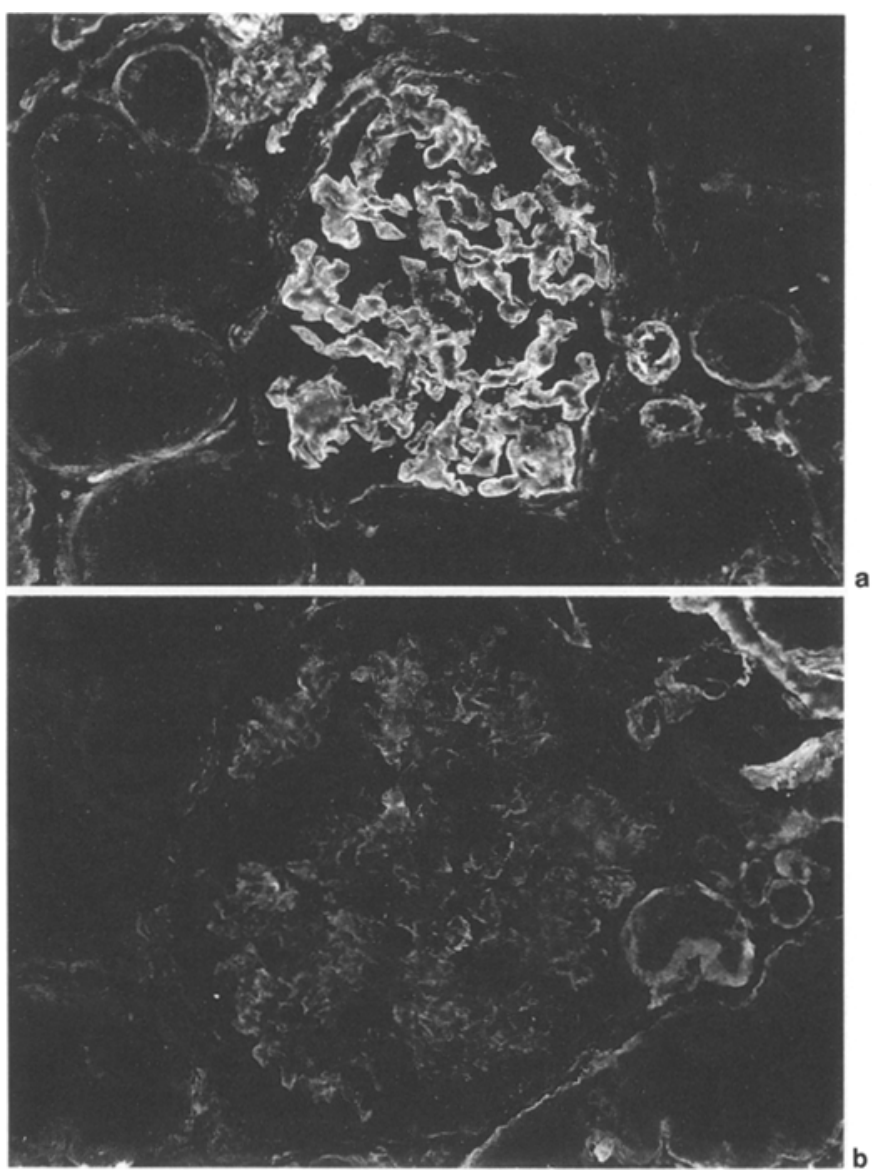

Fig.1 a,b. Control kidney section a, stained with a monoclonal antibody directed against HS (JM403). A linear staining pattern of high intensity is observed in the GBM (magnification $\times 250$ ). b, Kidney section of a patient with diabetic nephropathy with mesangial expansion without nodular lesions stained with the same anti-HS antibody. Compared to a the intensity of staining of HS chains is clearly diminished. Residual staining is occasionally seen (magnification $\times 250$ )

bumin in phosphate buffered saline (PBS) and incubated for $60 \mathrm{~min}$. The second layer was applied using the antibodies as described before. To prevent quenching p-phenylenediamine in PBS-glycerol was applied, the slides were scored by all observers within a short period, and due to the experience of the observers, the exposure to the fluorescence microscope lamp was kept to a minimum. There was no correlation between the order of the different observations and the intensity score, which indicates that quenching of the immunofluorescence signal did not influence the scoring.

Scoring procedure. For logistic reasons, the study was performed in four study periods. During each period, two control subjects and six patients with diabetes were analysed. The slides were numbered randomly in order to be examined without knowledge of the sample origin and were scored by four independent observers. The scoring was performed following standard procedures. Thus, a mean score was given for section assessed, expressing a representative score for the glomeruli present. The intensity of staining was scored on a semiquantitative scale: $0=$ negative, $1 / 2=$ slightly positive, $1=$ positive, $2=$ positive to strongly positive, $3=$ strongly positive. The staining pattern was described using the following terms: linear, fine granular, granular, discontinuous, irregular. 


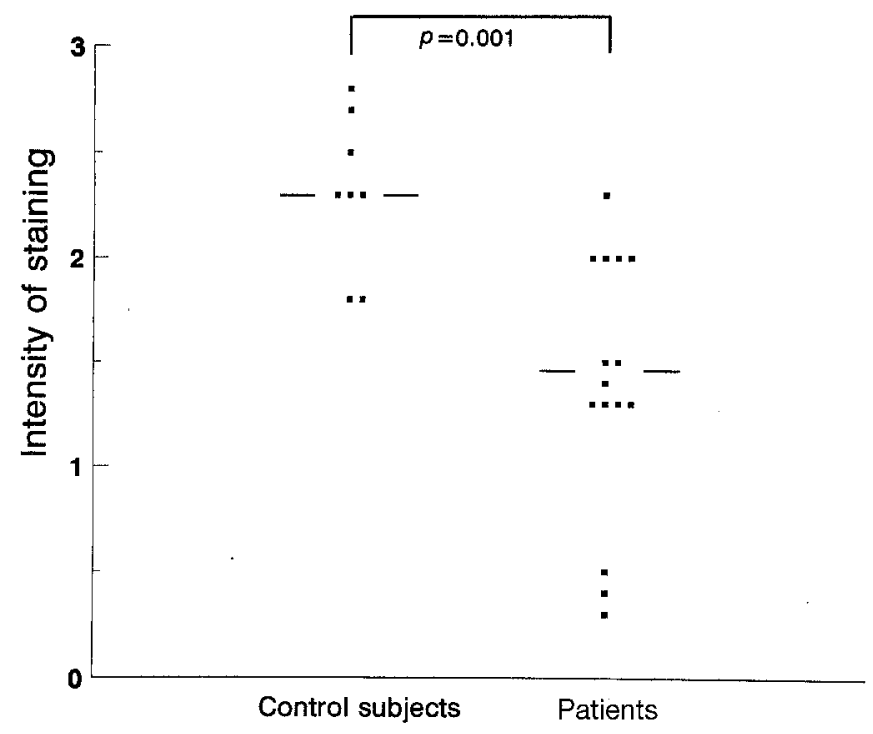

Fig. 2. GBM HS staining intensity of the control group vs the patients with diabetic nephropathy. The scores after staining with monoclonal antibody JM403 are depicted for each biopsy

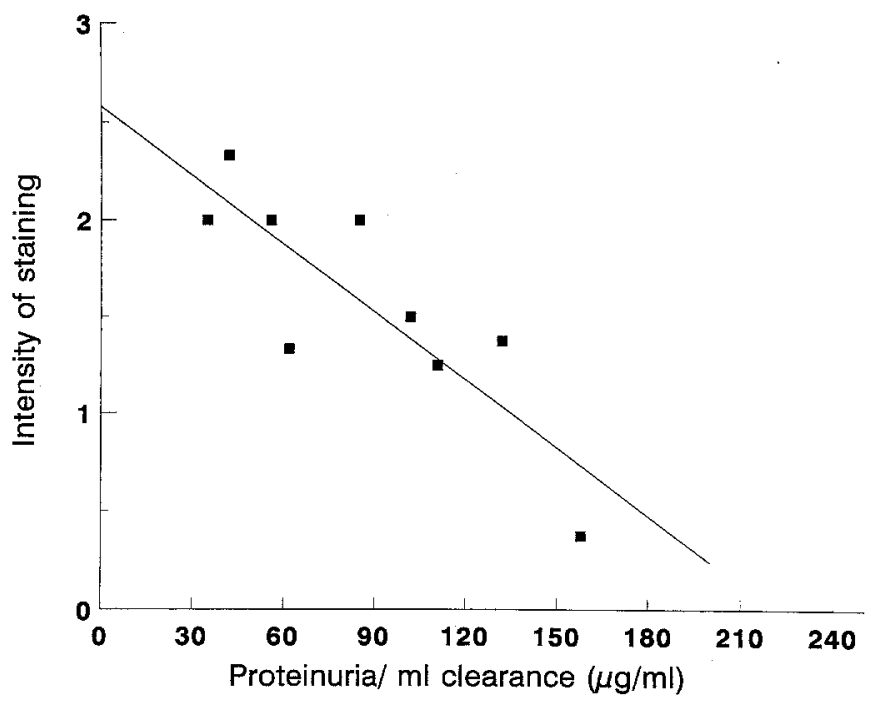

Fig. 3. GBM HS staining intensity vs proteinuria/ml creatinine clearance (see Methods). Staining intensity is depicted as mean of four observers. The equation of the regression line: $y=2.6+0.012 * x ; r=-0.85, p=0.004, n=9$. The intercept falls within the $95 \%$ confidence interval of the control subjects

\section{Statistical analysis}

Findings of the observers were compared using Pearson's product sum correlations. Agreement was defined as the percentage of corresponding scores. A deviation of one class in scoring intensity was accepted. Using this model, weighted Cohen's kappa were calculated. Mean scores and standard deviations were calculated for the control and diabetic groups, as were scoring differences (mean intensity score of the diabetic-minus the control group). Student's two sample $t$-test was used to determine $p$ values and $95 \%$ confidence intervals of the mean scoring difference. Test results with the Mann Whitney test were very similar. Spearman's rank sum correlation was used to determine correlation, and the regression of HS and collagen IV staining vs proteinuria was calculated.

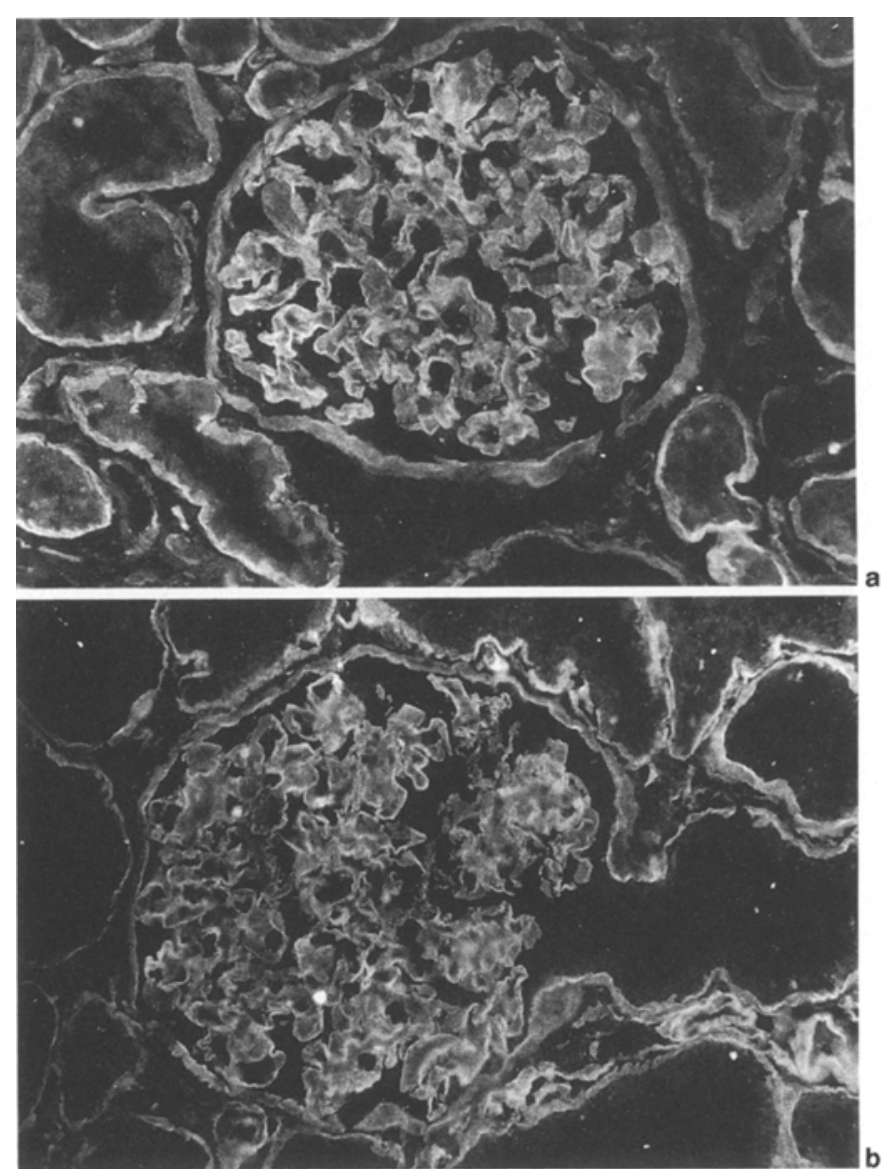

Fig.4a,b. Control kidney section a, stained with antibody B31 directed against HSPG-core protein (magnification $\times 250$ ). $\mathbf{b}$, In a diabetic glomerulus staining intensity and pattern are not altered (magnification $\times 250$ )

\section{Results}

In all observer combinations a highly significant correlation $(p<0.001)$ was observed for the biopsy scores, the correlation coefficients ranging from 0.66 to 0.80 . Cohen's weighted kappas ranged from 0.56 to 0.78 , the agreement in scoring from 77 to $89 \%$ (Table 3). Diabetic glomerulopathy was diagnosed in all 14 renal biopsies selected. Of 14 tissue sections with diabetic glomerulopathy 10 showed mesangial sclerosis with nodular lesions, in four, no nodular lesions were observed. Four patients had entered the stage of (pre)terminal renal insufficiency defined as a calculated creatinine clearance below $15 \mathrm{ml} / \mathrm{min}$. Data concerning retinopathy and proteinuria are shown in Table 1.

\section{Glomerular basement membrane}

$H S$ and HSPG-core protein. The kidney sections of the control group revealed staining of high intensity ( 2 to 3 ) with the anti-HS antibody JM403 (Fig. 1 A). The mean staining intensity of the control group was calculated to be 2.31 (Table 4). In the diabetic group (Fig. 1B) the in- 
Table 4. Intensity of staining presented as the mean (SD) of four observers

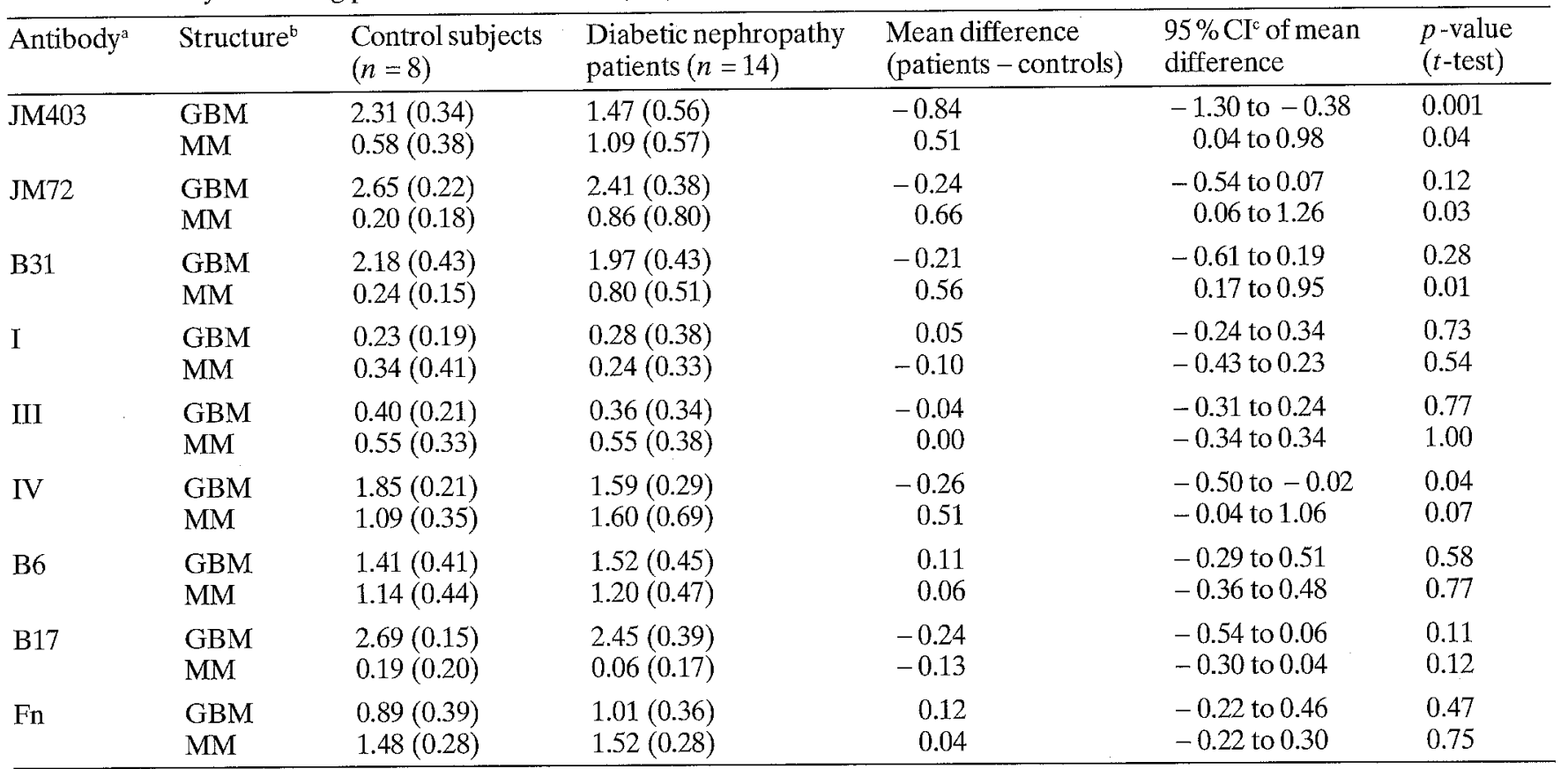

${ }^{\mathrm{a}}$ Codes of the antibodies as depicted in Table 2. ${ }^{\mathrm{b}} \mathrm{MM}$, mesangium. ${ }^{\mathrm{c}} \mathrm{CI}$, confidence interval

tensity ranged from 0 to 2 , with a mean intensity of 1.47 . The anti-HS immunofluorescence was significantly $(p=0.001)$ decreased in sections from patients with diabetic nephropathy (Fig. 2). The difference between the group means amounted to -0.84 (about one point on the scoring scale). In the control group a linear to fine granular staining pattern was observed (Fig. 1A). In the diabetic group the pattern varied from linear to granular to a discontinuation of the linearity (Fig. 1B). In the diabetic group HS staining intensity revealed a significant inverse correlation $(r=-0.85, p=0.004)$ with proteinuria corrected for creatinine clearance in the patients who had not entered the stage of preterminal renal insufficiency (Fig. 3). With anti-HSPG-core protein antibodies (JM72,B31), linear staining of the GBM was seen in both the diabetic and the control group (Fig. 4). The intensity ranged from 2 to 3 in both groups and no significant changes were noticed between the two groups (Table 4).

Collagens type IV, $\alpha 1(I V) N C$ and $\alpha 3(I V) N C$. Intensity of GBM staining was slightly but significantly decreased in diabetic nephropathy (Table $4, p=0.04$ ) for collagen type IV. When the subgroup with nodular sclerosis was analysed separately, the decrease in collagen IV in the GBM was more marked $(p=0.006)$. With anti- $\alpha 1$ (IV)NC (antibody B6) the difference in staining intensity between the two groups did not reach statistical significance (Table 4). The GBM showed a strongly positive reactivity with anti- $\alpha 3$ (IV)NC (antibody B17) in both the control and the diabetic group. No correlation was observed between collagen IV staining and proteinuria $(r=0.26, p=0.5)$.
Collagens type I, type III, and fibronectin. No anti-collagen type I activity was observed in the control and the diabetic group. Bowman's membrane and fibrous formations located directly below it showed intensive staining ( 2 to 3 ) in 9 of the 14 biopsies in the diabetic group. Antibodies to collagen type III showed no staining or occasionally slight positive staining in both the control and the diabetic group. A linear, frequently discontinuous staining of moderate intensity was observed in the GBM after staining of fibronectin. No differences were found between the diabetic and control group (Table 4).

\section{Mesangium (Table 4)}

$H S$ and HSPG-core protein. In the control group no or only very weak staining for the $\mathrm{HSPG}$-core protein was observed in the mesangium. In diabetic biopsies, HSPG-core protein staining was significantly increased, ranging from negative to strongly positive staining (Table 4). Biopsies with nodular sclerosis revealed a more marked increase of core protein staining. With anti-HS, staining ranged from 0 to 3 in the diabetic group, while negative or slightly positive staining was seen in the control subjects (Table 4).

Collagen type IV, $\alpha I(I V) N C$ and, $\alpha 3(I V) N C$. The results for collagen IV ranged from 0 to 3 in both the control and the diabetic patients. A significant $(p=0.003)$ increase of mesangial staining was observed for collagen type IV in biopsies with nodular sclerosis. Staining of $\alpha 1(\mathrm{IV}) \mathrm{NC}$ was positive in mesangial areas of 
controls and diabetic patients, while anti- $\alpha 3$ (IV)NC was negative in both groups.

Collagen type I, type III and, fibronectin. In both groups absent or slightly positive staining of the mesangial area was observed with anti-collagen type I antibodies. Infrequently, positive staining with anti-collagen type III was seen in nodular lesions. Fibronectin was abundantly present in the mesangial areas of several diabetic patients.

\section{Discussion}

We have demonstrated a decreased staining of HS in the GBM of patients with diabetic nephropathy, while staining of HSPG-core protein was normal. This decreased HS staining inversely correlated with the proteinuria/creatinine clearance ratio.

The scoring of the glomeruli was performed using standard procedures. Three sections had to be excluded because they were too small to be evaluated properly. Of the remaining sections, one contained only five open glomeruli. Excluding this biopsy would have slightly improved the difference between the two groups. Several measures were taken to prevent quenching and to confirm the reproducibility of the immunofluorescence signal. In short, tissue preparation was similar for each section, and carried out by the same skilled laboratory technician. The slides were covered with p-phenylenediamine, stored at $4{ }^{\circ} \mathrm{C}$, and scored within a short time period without extensive exposure of the slides to light. No differences in scoring of the signal intensity was noticed between the different observers, indicating that quenching had been limited and did not influence the outcome of the study. Furthermore, all biopsies were studied using a range of antibodies, which served as an internal control. For example, staining of HS was decreased in the biopsies with diabetic glomerulopathy, while in the same biopsies staining of HSPG-core protein was unaltered, suggesting an altered staining of HS only.

In contrast to most other immunofluorescence studies, we used four different observers who scored the slides without knowledge of the sample origin. The percentage of agreement among the observers was high, providing evidence that the results are not obscured by personal bias and indicating the reproducibility of the scoring procedure. Furthermore, the results obtained in this study were very similar to previously conducted pilot experiments in 12 patients with diabetic nephropathy and four control subjects. Three patients were studied both in the pilot and in the present study, and showed consistent results on both occasions. The consistency of the differences between the control subjects and patients with diabetic nephrópathy was illustrated by the fact that in each of the four study periods, similar results were obtained.
The decrease in HS staining was observed in patients with either IDDM or NIDDM. Analysis of the subgroups was not performed because of the small numbers of patients and the retrospective nature of the data. Retinopathy was reported to be absent in three NIDMM patients with biopsy proven diabetic nephropathy. Diabetic nephropathy without signs of retinopathy has been reported previously in patients with NIDDM [25]. Furthermore, as retinopathy was assessed by routine funduscopy, it may have gone undetected in some patients.

The decrease in HS staining could be explained by loss of the epitope, which may reflect an alteration in or decrease of HS molecules. Using biochemical and cytochemical methods HS has been observed to be reduced in human diabetic kidneys [3,11]. However, an antibody with specificity against the HS side chain was not available in earlier immunohistochemical studies. We were able to confirm the biochemical results and to document and visualize the HS decrease immunohistochemically.

Another explanation for the decreased HS staining may be an altered antibody-epitope interaction due to non-enzymatic glycation of extracellular matrix proteins [26]. HS itself is not a target of glycation, because it is composed of repeating disaccharide units. By contrast, extracellular matrix proteins surrounding and interacting with HS may be subjected to non-enzymatic glycation and thus change the extracellular matrix structure by crosslinking. Crosslinking could lead to a reduced accessibility of the epitope recognized by the anti-HS monoclonal antibodies. We did not observe differences in GBM HSPG-core protein staining between the diabetic and control group, which suggests a normal epitope antibody interaction in the protein part of the HSPG molecule, which is conceivably more susceptible to non-enzymatic glycation than HS epitopes. Thus, non-enzymatic glycation does not provide a very likely explanation for the decrease of HS staining.

GBM HSPG-core protein staining was not diminished in the diabetic group in our study. This finding confirmed an earlier immunohistochemical study [27]. We observed an increase in HSPG-core protein expression in the mesangial area which was most marked in biopsies with nodular lesions. This observation is in contrast to an earlier study [28] which reported a decreased HSPG-core protein staining in the glomerular matrix, using antibodies raised against porcine-HSPG. The difference may be due to the fact that our antihuman GBM HSPG antibodies recognize different epitopes on the HSPG-core proteins.

Decrease of HS staining in the GBM is not unique for diabetic nephropathy. In lupus nephritis, membranous nephropathy and minimal change disease, staining of HS was found to be decreased, while it was unaltered in IgA nephropathy and Alport's syndrome [18]. This suggests that diminished HS staining cannot be explained by proteinuria alone and the significant inverse 
correlation between the proteinuria-creatinine clearance ratio and the GBM anti-HS staining intensity may well represent a functional relationship between GBM HS loss and proteinuria.

HS may have a role in the pathogenesis of diabetic glomerulopathy. Metabolic factors such as glucose and insulin-like growth factor I lead to decreased mesangial HS production $[5-7,9,11,29]$ and changes in the chemical structure of HS $[4,5,8]$. In normal physiology, HS has been shown to be of importance for the integrity of the glomerular size- and charge barrier [13, 17]. Loss of GBM HS resulted in an increased permeation of albumin [13]. Thus, a decrease or change of HS could be involved in the pathophysiology of albuminuria, an early feature of diabetic nephropathy [30-33]. Furthermore, HS is able to inhibit mesangial proliferation $[15$, $16]$ and loss of HS may contribute to mesangial expansion, the histologic hallmark of diabetic glomerulopathy which correlated most strongly with clinical parameters [34]. Recently, a significant correlation between the decrease of GBM anionic sites and mesangial expansion has been described [11], compatible with the hypothesis that reduction of GBM HS might induce a compensatory increase in the synthesis of matrix components [35]. Further evidence of a role for HS has been provided by a study which showed that treatment of diabetic rats with sulphated glycosaminoglycan chains, completely prevented a loss of anionic sites, GBM thickening, and albuminuria [36]. Thus, loss of HS may have pathogenetic relevance in albuminuria and mesangial expansion, two important features of diabetic nephropathy.

The diabetes-induced glomerular changes do not only influence HS [24, 25, 37-39]. We stained our biopsies with antibodies reactive with fibronectin and several types of collagen. The more intense fibronectin staining of the mesangial area in some biopsies may be due to an expansion of the mesangium. Anti-collagen type III reactivity was rarely observed in diabetic nodules, confirming earlier observations in diabetic nephropathy and its experimental models $[27,40]$. It illustrates either a shift in phenotypic expression of glomerular cells or an invasion of collagen type III in an advanced diabetic glomerulopathy.

GBM collagen type IV staining was decreased which may be due to a decrease in matrix content of the GBM or to a decrease in concentration of the epitope. Increased expression of collagen type IV in the GBM is well known in diabetes and present early in diabetic nephropathy [38]. However, decreased expression of collagen type IV has been reported in advanced stages $[28,38,39]$. The decreased staining we observed indicates that the diabetic glomerulopathy was advanced in the majority of patients studied. No change in staining intensity of the GBM could be observed with a monoclonal antibody specific [24] to human anti- $\alpha 1$ (IV)NC. The polyclonal anti-collagen type IV antibody was raised against pepsin digested bovine and human placental collagen, and is thus most likely reactive against epitopes of the triple helical and the $7 \mathrm{~S}$ domains. The discrepancy of staining of our antibodies directed against classic collagen type IV epitopes confirms the hypothesis that the turnover of collagen type IV subchains are regulated separately in diabetic nephropathy. The brilliant GBM staining with anti- $\alpha 3$ (IV)NC antibodies in diabetic biopsies with nodular lesions which had lost anti-collagen type IV staining has been previously demonstrated [39]. It suggests that the decreased GBM matrix content may be joined by qualitative changes in collagen IV expression.

The most interesting finding of this study was the decreased expression of GBM HS without changes in HSPG-core protein staining in diabetic nephropathy. Future studies are needed to correlate changes of structural parameters with clinical parameters such as albuminuria, hypertension, duration of diabetes and metabolic control. The intriguing question of whether HS expression is selectively decreased in patients with microalbuminuria remains to be elucidated.

Acknowledgements. We thank Dr. P. E. de Jong, University Hospital Groningen, Dr. W. Geerlings, Martini Hospital Groningen, Dr. B.L.Hogewind, Bronovo Hospital The Hague, Dr. Y.I.Tjandra, Red Cross Hospital The Hague for kindly providing clinical data, Dr. L. P.W.J. van den Heuvel for producing the polyclonal antibody B31 and, Dr. N.A.T.Hamdy for carefully reading the manuscript.

\section{References}

1. Deckert T, Feldt-Rasmussen B, Borch-Johnsen K, Jensen T, Kofoed-Enevoldsen A (1989) Albuminuria reflects widespread vascular damage. The Steno hypothesis. Diabetologia 32: $219-226$

2. Kanwar YS, Rosenzweig LJ, Linker A, Jakubowski ML (1983) Decreased de novo synthesis of glomerular proteoglycans in diabetes: biochemical and autoradiographic evidence. Proc Natl Acad Sci USA 80: 2272-2275

3. Parthasarathy N, Spiro RG (1982) Effect of diabetes on the glycosaminoglycan component of the human glomerular basement membrane. Diabetes 31: 738-741

4. Cohen MP, Sarma MC (1981) $\left({ }^{35} S\right)$ Sulphate incorporation into glomerular basement membrane glycosaminoglycan is decreased in experimental diabetes. J Lab Clin Med 98: 715-722

5. Brown DM, Klein DJ, Michael AF, Oegema TR (1982) ${ }^{35} \mathrm{~S}-$ glycosaminoglycan and ${ }^{35}$ S-glycopeptide metabolism by diabetic glomeruli and aorta. Diabetes 31: 418-425

6. Kanwar YS, Rosenzweig LJ, Linker A (1983) Decreased de novo synthesis of glomerular proteoglycan in diabetes: biochemical and autoradiographic evidence. Proc Nat Acad Sci USA 80: 2272-2275

7. Rohrbach DH, Wagner CW, Star VL, Martin GR, Brown KS (1983) Reduced synthesis of basement membrane heparan sulphate proteoglycan in streptozotocin-induced diabetic mice. J Biol Chem 258: 11672-11677

8. Rohrbach DH, Hasell JR, Kleinman HK, Martin GR (1982) Alterations in the basement membrane (heparan sulphate) proteoglycan in diabetic mice. Diabetes $31: 185-188$ 
9. Wu VY, Wilson B, Cohen MP (1987) Disturbances in glomerular basement membrane glycosaminoglycans in experimental diabetes. Diabetes 36: 679-683

10. Klein D, Oegema TR, Brown DM (1989) Release of glomerular heparan ${ }^{35} \mathrm{SO}_{4}$ proteoglycan by heparin from glomeruli of streptozotocin-induced diabetic rats. Diabetes 38: 11301139

11. Vernier RL, Steffes MW, Sisson-Ross S, Mauer SM (1992) Heparan sulphate proteoglycan in the glomerular basement membrane in type 1 diabetes mellitus. Kidney Int 41: 10701080

12. Edge ASB, Spiro RG (1990) Characterization of novel sequences containing 3-O-sulphated glucosamine in glomerular basement membrane heparan sulphate and localization of sulphated disaccharides to a peripheral domain. J Biol Chem 265: 15874-15881

13. Rosenzweig LJ, Kanwar YS (1982) Removal of sulphated (heparan sulphate) or non sulphated (hyaluron acid) glycosaminoglycans results in increased permeability of the glomerular basement membrane to ${ }^{125}$ I albumin. Lab Invest 47: 177-184

14. Kanwar YS, Linker A, Farquhar MG (1980) Increased permeability of the glomerular basement membrane to ferritine after removal of glycosaminoglycans (heparan sulphate) by enzyme digestion. J Cell Biol 86: 688-693

15. Groggel GC, Marinides GN, Hovingh P, Hammond E, Linker A (1990) Inhibition of rat mesangial cell growth by heparan sulphate. Am J Physiol 258: F259-F265

16. Castellot JJ, Hoover RL, Harper PA, Karnovsky MJ (1985) Heparin and glomerular epithelial cell-secreted heparin-like species inhibit mesangial-cell proliferation. Am J Path 120: $427-435$

17. Born J van den, Heuvel LPWJ, Bakker MAH, Veerkamp JH, Assmann KJM, Berden JHM (1992) A monoclonal antibody against GBM heparan sulphate induces an acute selective proteinuria in rats. Kidney Int 41: 115-123

18. Born J van den, Heuvel LPWJ van den, Bakker MAH et al. (1993) Evaluation of the distribution of GBM heparan sulphate proteoglycan core protein and side chains in human glomerular diseases by monoclonal antibodies. Kidney Int 43

19. Fajans SS (1990) Classification and diagnosis of diabetes. In: Rifkin H, Porte D $\mathrm{J}_{\mathrm{r}}$ (eds) Diabetes mellitus: theory and practice. Elsevier, New York, pp 346-356

20. Østerby R, Parving H-H, Nyberg G et al. (1988) A strong correlation between glomerular filtration rate and filtration surface in diabetic nephropathy. Diabetologia 31: 265-270

21. Harris RD, Steffes MW, Bilous RW, Sutherland DER, Mauer SM (1991) Global glomerular sclerosis and glomerular arteriolar hyalinosis in insulin dependent diabetes. Kidney Int 40: 107-114

22. Van den Heuvel LPWJ, Van den Born J, Van de Velden TJAM et al. (1989) Isolation and partial characterization of heparan sulphate proteoglycan from the human glomerular basement membrane. Biochem J 264: 457-465

23. Bergijk EC, Munaut C, Baelde JJ et al. (1992) A histological study of the extracellular matrix during the development of glomerulosclerosis in murine chronic graft-versus-host disease. Am J Path 140: 1147-1156
24. Johansson C, Butkowski R, Wieslander J (1991) Characterization of monoclonal antibodies to the globular domain of collagen IV. Connect Tissue Res 25: 229-241

25. Parving HH, Gall MA, Sk $\phi t t$ P et al. (1992) Prevalence and causes of albuminuria in non-insulin-dependent diabetic patients. Kidney Int 41: 758-762

26. Brownlee M (1990) Advanced products of nonenzymatic glycosylation and the pathogenesis of diabetic complications. In: Rifkin H, Porte D Jr (eds) Diabetes mellitus: theory and practice. Elsevier, New York, pp 279-291

27. Suzuki Y (1989) Constituents of the extracellular matrices in diabetic glomerulosclerosis. Jap J Nephr 31: 47-54

28. Nerlich A, Schleicher E (1991) Immunohistochemical localization of extracellular matrix components in human diabetic glomerular lesions. Am J Path 139: 889-899

29. Moran A, Brown DM, Kim Y, Klein DJ (1991) Effects of IGF-1 and glucose on protein and proteoglycan synthesis by human fetal mesangial cells in culture. Diabetes 40: 1346 1354

30. Mogensen CE (1988) Definition of diabetic renal disease in insulin-dependent diabetes mellitus based on renal function test. In: Mogensen CE (ed) The kidney and hypertension in diabetes mellitus. Martinus Nijhoff Publishing, Boston, pp 7-16

31. Viberti GC, Jarrett RJ, Mahmud U, Hill RD, Argyropoulos A, Keen H (1982) Microalbuminuria as a predictor of clinical nephropathy in insulin dependent diabetes mellitus. Lancet I: $1430-1432$

32. Mogensen CE, Christensen CK (1984) Predicting diabetic nephropathy in insulin-dependent patients. N Engl J Med 311: 89-93

33. Mathiesen ER, Oxenbøll B, Johansen K, Svendsen PA, Deckert T (1984) Incipient nephropathy in type 1 (insulindependent) diabetes. Diabetologia 26: 406-410

34. Mauer SM, Steffes MW, Ellis EN, Sutherland DER, Brown DM, Goetz FC (1984) Structural-functional relationships in diabetic nephropathy. J Clin Invest 74: 1143-1155

35. Deckert T, Feldt-Rasmussen B, Mathiesen ER (1984) Pathogenesis of diabetic nephropathy: a hypothesis. Diabetic Nephropathy 3: 83-88

36. Gambaro G, Cavazzana AO, Luzi P et al. (1992) Glycosaminoglycans prevent morphological renal alterations and albuminuria in diabetic rats. Kidney Int 42: 285-291

37. Shimomura H, Spiro RG (1987) Studies on macromolecular components of human glomerular basement membrane and alterations in diabetes. Diabetes 36: 1374-1381

38. Falk RJ, Scheinman JI, Mauer SM, Michael AF (1983) Polyantigenic expansion of basement membrane constituents in diabetic nephropathy. Diabetes 32 [Suppl 2]: 34-39

39. Kim Y, Kleppel MM, Butkowski R, Mauer SM, Wieslander J, Michael AF (1991) Differential expression of basement membrane collagen chains in diabetic nephropathy. Am J Path 138: 413-420

40. Abrass CK, Peterson CV, Raugi GJ (1988) Phenotypic expression of collagen types in mesangial matrix of diabetic and nondiabetic rats. Diabetes 37: 1695-1702

41. L'Esperance FA Jr, James WA, Judson PH (1990) The eye and diabetes mellitus. In: Rifkin H, Porte D Jr (eds) Diabetes mellitus: theory and practice. Elsevier, New York, pp 661683 